\title{
Treatment of In-Stent Stenosis Following Flow Diversion of Intracranial Aneurysms with Cilostazol and Clopidogrel
}

\author{
Ehsan Dowlati, $\mathrm{MD}^{1}$, Kory B. Dylan Pasko, BS ${ }^{2}$, Jiaqi Liu, BS ${ }^{2}$, Charles A. Miller, MD³, \\ Daniel R. Felbaum, $\mathrm{MD}^{1,4}$, Samir Sur, $\mathrm{MD}^{1,4}$, Jason J. Chang, $\mathrm{MD}^{5}$, Ai-Hsi Liu, $\mathrm{MD}^{6}$, \\ Rocco A. Armonda, $\mathrm{MD}^{1,4}$, Jeffrey C. Mai, $\mathrm{MD}, \mathrm{PhD}^{1,4}$ \\ ${ }^{1}$ Department of Neurosurgery, MedStar Georgetown University Hospital, Washington, DC, USA \\ ${ }^{2}$ Georgetown University School of Medicine, Washington, DC, USA \\ ${ }^{3}$ Division of Neurosurgery, Walter Reed National Military Medical Center, Bethesda, MD, USA \\ ${ }^{4}$ Department of Neurosurgery, MedStar Washington Hospital Center, Washington, DC, USA \\ ${ }^{5}$ Department of Critical Care Medicine, MedStar Washington Hospital Center, Washington, DC, USA \\ ${ }^{6}$ Department of Radiology, MedStar Washington Hospital Center, Washington, DC, USA
}

\begin{abstract}
In-stent stenosis is a feared complication of flow diversion treatment for cerebral aneurysms. We present 2 cases of patients treated with pipeline flow diversion for unruptured cerebral aneurysms. Initial perioperative dual antiplatelet therapy (DAPT) consisted of standard aspirin plus clopidogrel. At 6-month follow-up cerebral angiography, the patients were noted to have developed significant in-stent stenosis (63\% and 53\%). The patients were treated with cilostazol and clopidogrel for at least 6 months. Subsequent angiography at 1-year post-treatment showed significant improvement of the in-stent stenosis from $63 \%$ to $34 \%$ and $53 \%$ to $21 \%$. The role of cilostazol as treatment of intracranial in-stent stenosis has not been previously described. Cilostazol's vasodilatory effect and suppression of vascular smooth muscle proliferation provides ideal benefits in this setting. Cilostazol plus clopidogrel may be a safe and effective alternative to standard DAPT for treatment of in-stent stenosis following flow diversion and warrants further consideration and investigation.
\end{abstract}

Key Words: Endovascular procedures; Intracranial aneurysm; Stents; Cilostazol; Clopidogrel; Aspirin.

\section{INTRODUCTION}

Flow diverters are used for aneurysm embolization and are currently approved by the Food and Drug Administration for treatment of unruptured supraclinoid internal carotid artery (ICA) aneurysms. Flow diverters are deployed endovascularly for treatment of intracranial aneurysms and act as a scaffold for endothelial re-establishment. ${ }^{1}$ However, the metallic surface presents an inherent risk for platelet activation and subsequent thrombus formation prior to complete endothelial reconstruction. ${ }^{2}$ As with other endovascular treatments, flow diversion incurs risks including hemorrhage, failure of complete treatment, and acute thromboembolic events. To mitigate the risks of thromboembolic complications, perioperative dual antiplatelet therapy (DAPT) is a cor-

\section{Correspondence to: Jeffrey C. Mai, MD, PhD Department of Neurosurgery, Med- Star Georgetown University Hospital, 3800 Reservoir Rd NW, PHC 7, Wash- ington, DC 20007, USA \\ Tel: +1-202-877-5111 \\ Fax: +1-202-444-7333 \\ E-mail: jeffreymai@gmail.com}

Received: June 10, 2021

Revised: July 19, 2021

Accepted: July 26, 2021
Copyright $\odot 2021$ Korean Society of Interventional Neuroradiology This is an Open Access article distributed under the terms of the Creative Commons Attribution Non-Commercial License (http://creativecommons.org/licenses/by-nc/4.0) which permits unrestricted non-commercial use, distribution, and reproduction in any medium, provided the original work is properly cited.

pISSN 2093-9043 eISSN 2233-6273 
nerstone of flow diversion medical management. First-line, standard DAPT for pipeline flow diversion typically consists of full-dose aspirin and clopidogrel. Despite standard DAPT use, current literature reports flow diversion thromboembolic complication rates between $4 \%$ and $9 \%{ }^{3,4}$ Additionally, instent stenosis has been reported in a number of case reports and case series with relatively high incidence. ${ }^{5-8}$

Alternative antiplatelet options to standard DAPT, including ticagrelor and prasugrel, have been investigated to mitigate the risks of thromboembolic complications in cerebral aneurysms treated with flow diversion. ${ }^{2}$ However, cilostazol has not been well studied in this setting. As a potential alternative, cilostazol has been shown to inhibit platelet aggregation while also simultaneously vasodilating and suppressing pathological proliferation of vascular smooth muscle. ${ }^{9}$ Therefore, we hypothesize that cilostazol and clopidogrel may prove beneficial for in-stent stenosis following pipeline flow diversion that is refractory to standard DAPT.

We present case illustrations of 2 patients with an unruptured ICA aneurysm that were treated with Pipeline Flex Embolization Device (PED; Medtronic, Irvine, CA, USA) for flow diversion. The subsequent development of in-stent stenosis was treated with cilostazol and clopidogrel following initial prophylactic treatment with standard aspirin plus clopidogrel. Subsequent digital subtraction angiography (DSA) demonstrated significant improvement in luminal narrowing following cilostazol treatment with resolution of in-stent stenosis. This is likely due to the unique ability of cilostazol to prevent platelet aggregation while simultaneously mitigating negative vascular remodeling. Although a causal relationship cannot be determined in these cases, cilostazol may have helped resolve in-stent stenosis and this warrants further investigation.

\section{CASE REPORT}

At our institution, we perform a routine 6-month follow-up DSA unless a patient becomes symptomatic, at which point we obtain a magnetic resonance angiogram (MRA) sooner. Following the 6-month DSA, another routine follow-up DSA is performed at 1-year. During these timepoints, $\mathrm{P} 2 \mathrm{Y} 12$ reaction unit testing is performed to confirm anti-platelet response. Thereafter, a follow-up MRA is obtained at 3-year and 5-year follow-up. If there is a concern for in-stent stenosis at any point in time, an MRA is obtained within 6 months.

We present a retrospective analysis of 2 patients who underwent PED for flow diversion treatment of an unruptured left supraclinoid ICA aneurysm and subsequently developed significant in-stent stenosis on follow-up DSA. A summary is noted in Table 1 and Fig. 1. Institutional Review Board approval was waived for this study. All patients provided written informed consent for this study.

\section{Case 1}

A 68-year-old female with hypertension, hyperlipidemia, peripheral vascular disease, and 1 pack-per-day smoking presented with transient ischemic attacks (TIA). She was neurologically intact on examination. An unruptured left ICA aneurysm was incidentally discovered during the workup of her TIA. Left paraclinoid ICA fusiform segmented aneurysm (10.8×8.6×9.1 mm in size) was visible on magnetic

\section{Table 1. Patient case summaries}

\begin{tabular}{lll}
\hline Variable & \multicolumn{1}{c}{ Case 1 } & Case 2 \\
\hline Aneurysm & $10.8 \mathrm{~mm}$ left ICA paraclinoid fusiform aneurysm & $5.1 \mathrm{~mm}$ left SHA aneurysm \\
Treatment & 3.75 by $16 \mathrm{~mm}$ pipeline flex embolization device & 4 by $14 \mathrm{~mm}$ pipeline flex embolization device \\
\hline 6-month follow-up & $63 \%$ in-stent stenosis & $53 \%$ in-stent stenosis \\
12-month follow-up & $34 \%$ in-stent stenosis & $21 \%$ in-stent stenosis \\
Medication regimen & Clopidogrel 75 mg QD & Clopidogrel 75 mg QOD \\
& Aspirin 325 mg QD $\rightarrow$ cilostazol 100 mg BID & Aspirin 325 mg QD $\rightarrow$ cilostazol 100 mg BID \\
PRU & 112 & 84 \\
Plan & Continue clopidogrel+cilostazol & Stop clopidogrel+cilostazol \\
& MRA at 18-month follow-up & Aspirin 325 mg QD \\
& & MRA at 24-month follow-up \\
\hline
\end{tabular}

PRU, P2Y12 reaction unit; ICA, internal carotid artery; QD, daily; BID, twice daily; MRA, magnetic resonance angiogram; SHA, superior hypophyseal artery; QOD, every other day. 
resonance imaging (MRI) and confirmed with DSA (Fig. 2A). The patient elected to be treated with PED flow diversion. A $3.75 \times 16 \mathrm{~mm}$ PED was successfully deployed in the left supraclinoid ICA across the aneurysm. Following the procedure, the patient was continued on standard aspirin (325 mg) plus clopidogrel $(75 \mathrm{mg}$ ) as DAPT. A post-procedure head computed tomography (CT) showed no new signs of ischemia or hemorrhage. The patient remained neurologically intact and was discharged after observation in the intensive care unit (ICU). Follow-up cerebral DSA at 6-months demonstrated interval development of significant in-stent stenosis of the left ICA with 63\% luminal narrowing $(1.99 \mathrm{~mm}$ diameter just distal to flow diverter vs. $0.74 \mathrm{~mm}$ in-stent). The degree of stenosis was measured as a ratio of the narrowest in-stent diameter and distal normal diameter of the supraclinoid ICA (Fig. 2B). The patient's DAPT regimen was switched at this time, and she was treated with cilostazol $100 \mathrm{mg}$ twice daily (BID) and clopidogrel 75 mg daily. At 1-year follow-up, repeat DSA of the left ICA demonstrated significant improvement of in-stent stenosis to 34\% luminal narrowing (2.17 mm just distal to flow diverter vs. $1.43 \mathrm{~mm}$ in-stent) (Fig. 2C). Given the residual stenosis and her smoking history, the patient planned to continue taking cilostazol and clopidogrel, obtain a follow-up head MRA with contrast in 1 year, and repeat the angiogram in 2 years. The patient experienced no adverse events from the medication and no thromboembolic or hemorrhagic complications during this follow-up period.

\section{Case 2}

A 38-year-old woman with a history of chronic migraines presented to the emergency department with worsening head and neck pain that was attributed to her migraine. She reported that over the last month, she had increasing left-sided headaches associated with photophobia and intermittent nausea. She had no neurological deficits. Upon work-up of her worsening migraine symptoms, an unruptured left superior hypophyseal ICA aneurysm, visible on MRI/MRA and confirmed with DSA, was measured to be $5.1 \times 4.3 \times 5.3 \mathrm{~mm}$. Subarachnoid hemorrhage was ruled out with a lumbar puncture negative for xanthochromia. After

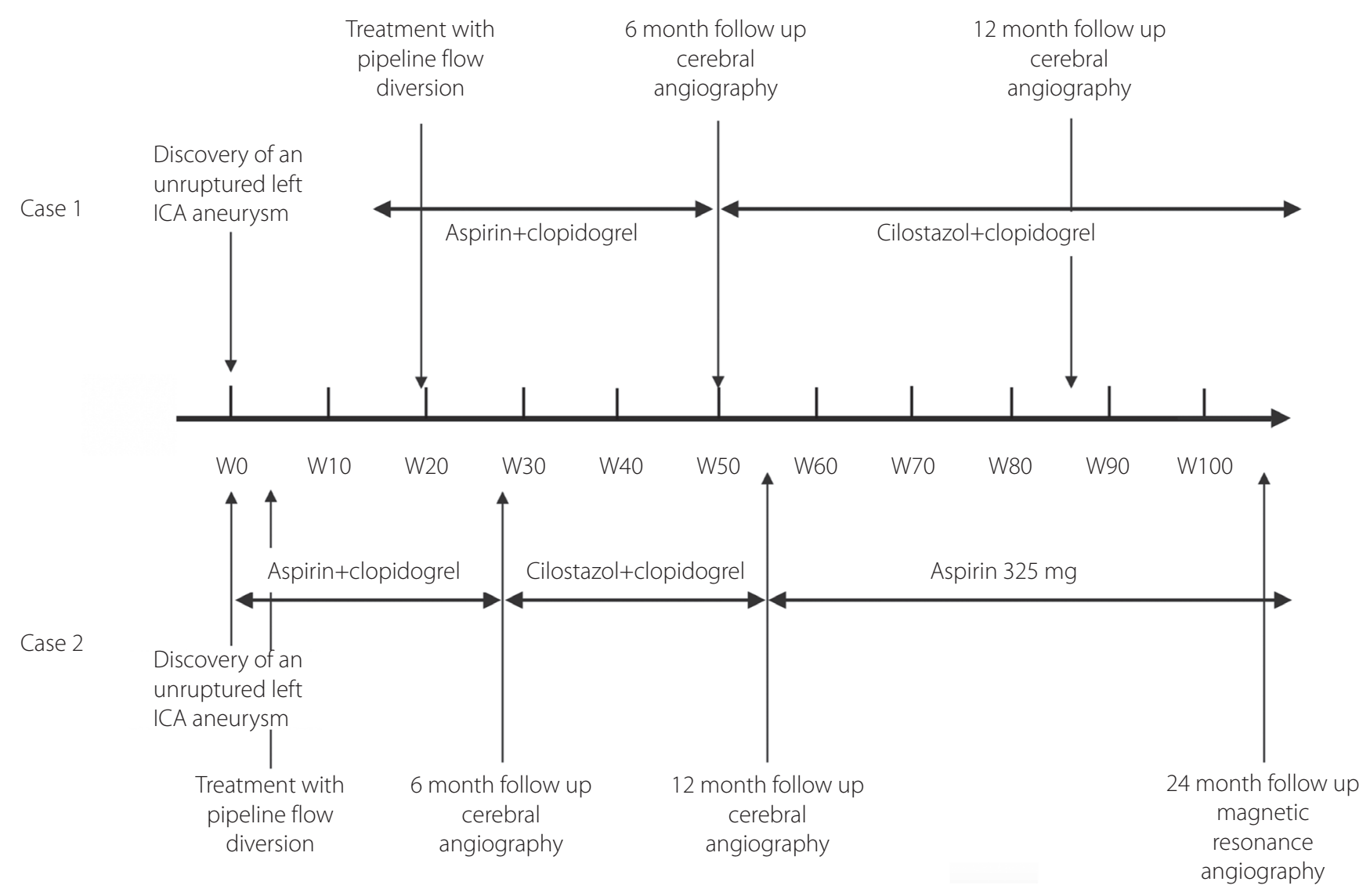

Fig. 1. Timeline of diagnosis and treatment of patient cases. ICA, internal carotid artery; Wo, discovery of aneurysm; W, week. 
discussion with the patient regarding the size of the aneurysm and rupture risk, the patient elected to be treated with PED flow diversion. The patient was pre-treated with aspirin $325 \mathrm{mg}$ and clopidogrel $75 \mathrm{mg}$ daily and was continued on DAPT following the procedure. A $4 \times 14 \mathrm{~mm}$ PED was deployed in the left supraclinoid ICA across the aneurysm (Fig. 3A). The patient remained neurologically intact and was discharged after observation in the ICU. Follow-up DSA at 6 months post-procedure demonstrated 53\% in-stent stenosis of the left ICA at the location of the flow diverter $(3.41 \mathrm{~mm}$ diameter of ICA just proximal to flow diverter vs. $1.60 \mathrm{~mm}$ ) (Fig. 3B). She was treated with cilostazol $100 \mathrm{mg} \mathrm{BID}$ and clopidogrel 75 mg every other day given a low P2Y12 reactivity unit result indicating that she was a hyper-responder. At 1-year follow-up, DSA demonstrated significant resolution of the in-stent stenosis down to $21 \%$ (3.42 mm proximal to flow diverter versus $2.69 \mathrm{~mm}$ at the narrowest segment in-stent) (Fig. 3C). Given the significant improvement of the in-stent stenosis, the patient's cilostazol and clopidogrel were discontinued at this point and she was maintained with aspirin $325 \mathrm{mg}$ daily monotherapy indefinitely. Her follow-up MRA at 2 years post-procedure showed no significant in-stent stenosis. There were no further adverse events during the follow-up period, and she tolerated her medications well without any thromboembolic or hemorrhagic events.

\section{DISCUSSION}

Our 2 cases demonstrated the potential efficacy of cilostazol and clopidogrel DAPT in treating in-stent stenosis after intracranial flow diversion. The pathophysiology of in-stent stenosis has been studied in pre-clinical models in the setting of cardiac and neurovascular intervention. It is hypothesized to be caused by thrombus development and inflammation leading to endothelialization and followed by late phase smooth muscle cell formation. ${ }^{6}$ Cilostazol's mechanism of action and pharmacology may help explain its utility in instent stenosis.

Classically, aspirin and P2Y12 receptor blockers, most commonly clopidogrel, are used as DAPT in flow diversion cases. Clopidogrel exerts its antiplatelet effect by irreversible inhibition of the P2Y12 adenosine diphosphate receptor and preventing expression of GPIIb/llla receptors on the platelet surface, thus blocking the aggregation and activation of platelets. $^{2}$ Since clopidogrel is a prodrug requiring CYP450 hepatic activation, its drug efficacy varies greatly depending on the responsiveness of the patient. Hypo-responders to clopidogrel have been reported to have higher risks of thrombotic complications, such as myocardial infarction, stroke, and cardiovascular death. ${ }^{10}$ Hyper-responders, on the other hand, are more likely to have hemorrhagic events. ${ }^{3}$ Prasugrel, an indirect P2Y12 irreversible inhibitor, acts similarly but utilizes a different activation pathway that includes rapid de-esterification prior to conversion of the active metabolite; therefore, its metabolism is not impacted by CYP genetic polymorphisms. ${ }^{10}$ Ticagrelor exerts its antiplatelet effect by reversible inhibition of P2Y12 receptors. Unlike clopidogrel, the direct-acting thienopyridine ticagrelor does not require hepatic activation, thus facilitating a more rapid and greater
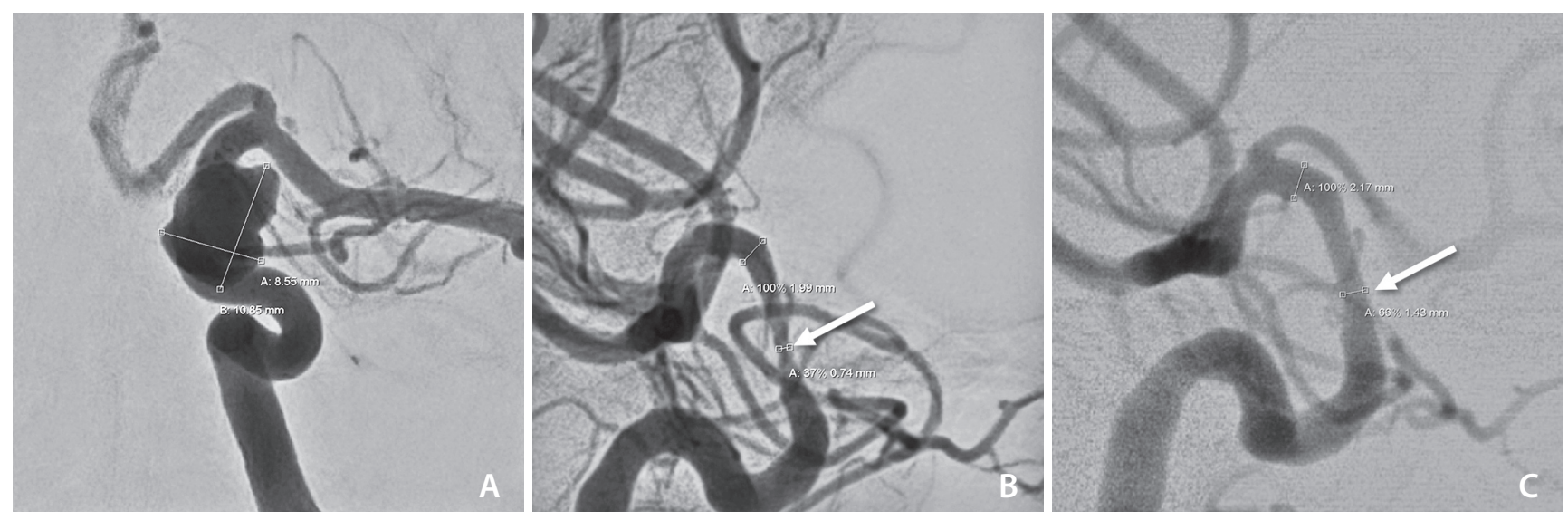

Fig. 2. Angiographic images for Case 1. (A) Left internal carotid artery (ICA) injection showing a left fusiform paraclinoid aneurysm measuring $8.5 \mathrm{~mm}$ by $10.8 \mathrm{~mm}$. (B) Six-month follow-up cerebral angiogram with left ICA injection showing significant in-stent stenosis of up to 63\% compared to immediate distal ICA diameter (white arrow). (C) Cerebral angiogram with left ICA injection at one-year follow-up from procedure and 6-month post cilostazol treatment demonstrating improved in-stent stenosis decreased to 34\% (white arrow). 
antiplatelet response. ${ }^{2}$ Studies suggest that DAPT regimens, which include ticagrelor or prasugrel, are safe and effective alternatives to clopidogrel-based DAPT regimens for prevention of thromboembolic complications in patients undergoing flow diversion procedures. ${ }^{11}$ However, the use of newer P2Y12 inhibitors like ticagrelor and prasugrel remains limited to special circumstances, such as clopidogrel resistance."

Cilostazol, on the other hand, exerts its effect by inhibiting phosphodiesterase IIIA (PDE-3A) activity, leading to increased levels of cyclic adenosine monophosphate (CAMP). Elevation in cAMP levels in blood vessels and platelets increase active forms of protein kinase $A$, which subsequently lead to direct inhibition of platelet aggregation, in addition to inducing vasodilatory effects on vascular smooth muscle and the ability to suppress vascular smooth-muscle cell proliferation, improve the function of vascular endothelium, and inhibit the pathological proliferation of the vascular intima. ${ }^{9}$ As such, the efficacy of cilostazol has been explored in treating neurovascular and cardiovascular diseases. In one study for ischemic stroke, cilostazol combined with aspirin reduced the risk of stroke recurrence compared to aspirin or clopidogrel alone. ${ }^{12}$ Cilostazol also prevents the progression of the symptomatic intracranial arterial stenosis due to its ability to suppress vascular smooth muscle proliferation and inhibition of pathologic proliferation of vascular intima. These unique properties of cilostazol may explain why our DAPT regimen of cilostazol and clopidogrel improved pipeline in-stent stenosis that proved to be refractory to standard DAPT.
While the use of cilostazol in intracranial neurovascular procedures as a means of mitigating thromboembolic complications remains limited, the effectiveness of cilostazol in reducing complications, such as in-stent stenosis, has been widely demonstrated in cardiovascular literature. Early studies of coronary artery restenosis rates following primary stenting in patients treated with standard DAPT were reported to be between 15 and $30 \% .^{13}$ As an alternative to standard DAPT, cilostazol was investigated and shown to have significantly lower rates of restenosis when used as an alternative to, or in conjunction with, clopidogrel as triple antiplatelet therapy. ${ }^{14}$ Douglas et al..$^{15}$ conducted a randomized, double-blind, placebo-controlled trial in 705 subjects after coronary artery stent placement and demonstrated significant improvement in restenosis rate in the cilostazol group compared to the placebo group.

Less frequent restenosis after cilostazol use has also been demonstrated in carotid artery stenting (CAS). Restenosis after CAS is noted in up to $8 \%$ of patients. ${ }^{16}$ Studies have shown that cilostazol use leads to decreased rates of in-stent restenosis and improved patency compared to other antiplatelet agents such as ticlopidine or clopidogrel. The results suggest this is due to the inhibitory effect on smooth muscle cell growth. ${ }^{17}$ In a meta-analysis of 6 studies, Galyfos et al. ${ }^{18}$ demonstrated significantly lower in-stent stenosis after CAS with cilostazol use without affecting other severe complication rates for myocardial infarction, stroke, or death. When patients are hypo-responders to clopidogrel, cilostazol has
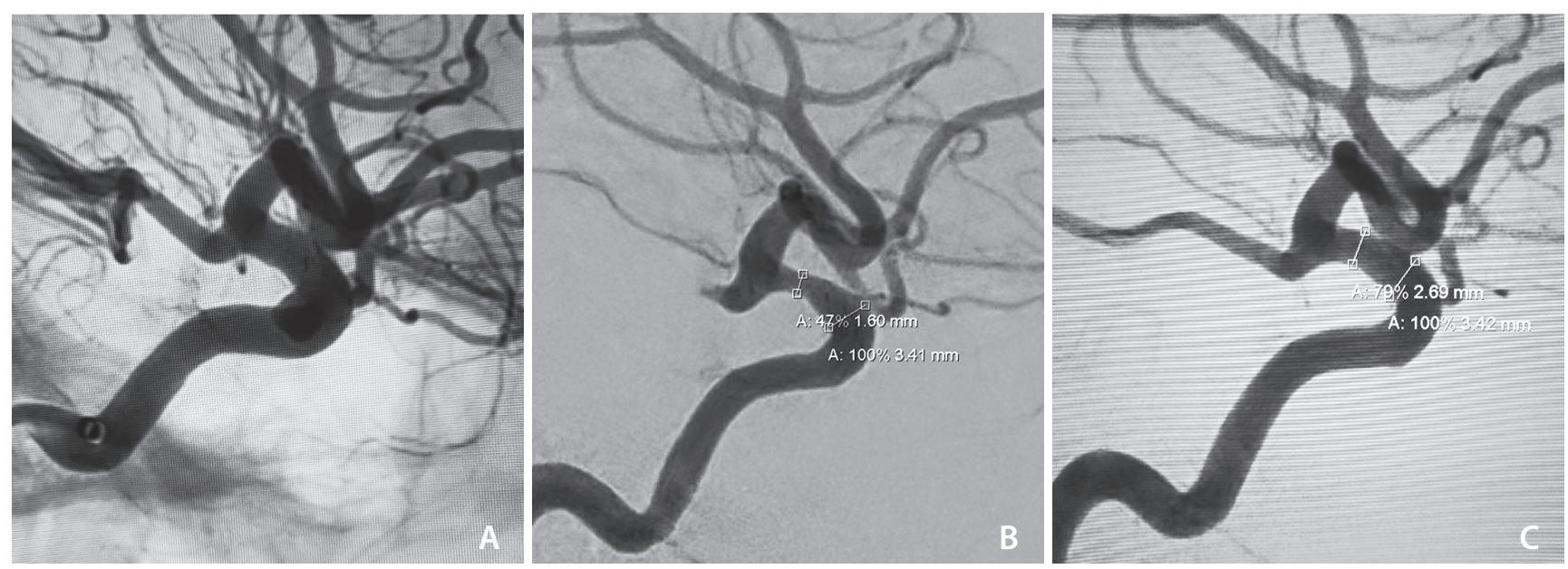

Fig. 3. Angiographic and imaging for Case 2. (A) Left ICA injection after Pipeline Embolization Device deployment showing normal ICA caliber without stenosis. (B) Six-month follow-up cerebral angiogram with left ICA injection showing significant in-stent stenosis of 53\% compared to immediate proximal ICA diameter. (C) One-year follow-up angiogram from the procedure and 6-month post cilostazol treatment demonstrating improved instent stenosis decreased to $21 \%$. 
been shown to be an effective option to decrease rates of ischemic events in patients with CAS. ${ }^{19}$

Although there is strong evidence to support the efficacy of cilostazol in treating cardiac endovascular intervention and $\mathrm{CAS}^{20}$ there is still limited evidence to strongly support cilostazol as a better candidate or adjunct in dual antiplatelet treatment for in-stent stenosis following flow diversion of intracranial aneurysms. While the extrapolation of data and treatment protocols from cardiac and vascular surgery may be helpful, it should not be done without a complete understanding of the risks and benefits. Future studies should be conducted to further explore the role of cilostazol in reducing thromboembolic complications seen in intracranial stents. In our patients, the adoption of cilostazol plus clopidogrel DAPT was only utilized after follow-up DSA showed worsening restenosis and treatment failure of standard DAPT.

Additionally, the challenge of determining clinically significant stenosis needs to be evaluated. Rates of in-stent stenosis following flow diversion are limited to case series and range from $9.8-39.0 \%{ }^{5,7,21}$ While a definitive time course of the cellular changes seen in humans after flow diversion has yet to be elucidated, studies utilizing animal models allow us to make general predictions of the time course of events causal for in-stent stenosis formation. The stages consist of inflammation and thrombus formation (0-30 days), endothelialization and granulation tissue (14-90 days), and smooth muscle formation and matrix formation (2-6 months). ${ }^{22}$ One study demonstrated a stenotic area with thrombus on the surface of the device struts, covered by neointima in histological preparations of flow diverters placed in pigs, which can provide a possible explanation for in-stent stenosis. ${ }^{23}$

The challenge is to determine which patient is at risk of becoming symptomatic from in-stent stenosis and what are the predisposing factors. Fortunately, the natural history of most in-stent stenosis appears to be benign and transient, ${ }^{6,7}$ but this conclusion is based on limited data influenced by a small number of case series and likely underreporting. It may be difficult to justify additional endovascular treatment, such as balloon angioplasty, for in-stent stenosis given the potential hemorrhagic complications of treating an already diseased segment compared to the reported natural history, ${ }^{5}$ but if cilostazol proves to be a useful adjunct in antiplatelet therapy in flow diversion then only the safety profile of the medication needs to be considered.

Finally, when using cilostazol, its side effects and contraindications should be considered. Cilostazol has been re- ported to increase cardiovascular mortality in patients who have congestive heart failure due to its PDE-3A inhibitory effects. ${ }^{24}$ It is, therefore, contraindicated in patients with heart failure. Lastly, the Medicare fee schedule cost of cilostazol is significantly higher than aspirin or clopidogrel for patients. Cost effectiveness has been established for use in patients with coronary stents. ${ }^{25}$ However, the cost-benefit of using cilostazol for intracranial stenting should be analyzed especially if financial constraints are suspected to present a barrier to individual patient care.

We present a use of cilostazol and clopidogrel as a possible alternative option to standard DAPT for treatment of intracranial in-stent stenosis following PED flow diversion with favorable results. The natural history of in-stent stenosis from flow diversion is not well elucidated and cilostazol may be a useful treatment option. Although we cannot claim a causal relationship based on this limited case series, cilostazol's ability to prevent platelet aggregation while simultaneously mitigating negative vascular remodeling events likely underlies the reversal of in-stent stenosis and prevention of thromboembolic complications observed in these 2 cases. Future comparative studies on the effect and mechanisms underlying cilostazol in preventing thromboembolic complications seen in intracranial aneurysms treated with flow diversion are warranted.

\section{Fund}

None.

\section{Ethics Statement}

Institutional Review Board approval was waived for this study. Informed consent for publication were obtained from all patients/parents.

\section{Conflicts of Interest}

The authors have no conflicts to disclose.

\section{Author Contributions}

Concept and design: ED, CM, and JM. Analysis and interpretation: ED, KP, JL, CM, SS, AL, JC, RA, and JM. Data collection: $E D, K P, J L, C M$, and DF. Writing the article: ED, KP, JL, and JM. Critical revision of the article: $E D, C M, D F, S S, A L, J C, R A$, and JM. Final approval of the article: ED, DF, SS, AL, JC, RA, and JM.

\section{ORCID}

Ehsan Dowlati: https://orcid.org/0000-0001-6739-0793 
Kory B. Dylan Pasko: https://orcid.org/0000-0001-8669-344X

Jiaqi Liu: https://orcid.org/0000-0003-2036-8200

Charles A. Miller: https://orcid.org/0000-0003-1893-5149

Daniel R. Felbaum: https://orcid.org/0000-0002-0156-4671

Samir Sur: https://orcid.org/0000-0003-4690-4921

Jason J. Chang: https://orcid.org/0000-0002-0825-3008

Ai-Hsi Liu: https://orcid.org/0000-0001-9883-4393

Rocco A. Armonda: https://orcid.org/0000-0002-8022-0411

Jeffrey C. Mai: https://orcid.org/0000-0002-4990-6431

\section{REFERENCES}

1. Fiorella D, Lylyk P, Szikora I, Kelly ME, Albuquerque FC, McDougall CG, et al. Curative cerebrovascular reconstruction with the Pipeline embolization device: the emergence of definitive endovascular therapy for intracranial aneurysms. J Neurointerv Surg 2018;10(Suppl 1):i9-i18

2. Tonetti DA, Jankowitz BT, Gross BA. Antiplatelet therapy in flow diversion. Neurosurgery 2020;86(Suppl 1):S47-S52

3. Daou B, Starke RM, Chalouhi N, Barros G, Tjoumakaris S, Rosenwasser $\mathrm{RH}$, et al. P2Y12 reaction units: effect on hemorrhagic and thromboembolic complications in patients with cerebral aneurysms treated with the pipeline embolization device. Neurosurgery 2016;78:27-33

4. Nelson PK, Lylyk P, Szikora I, Wetzel SG, Wanke I, Fiorella D. The pipeline embolization device for the intracranial treatment of aneurysms trial. AJNR Am J Neuroradio/ 2011;32:34-40

5. Cohen JE, Gomori JM, Moscovici S, Leker RR, Itshayek E. Delayed complications after flow-diverter stenting: reactive in-stent stenosis and creeping stents. J Clin Neurosci 2014;21:1116-1122

6. Mühl-Benninghaus R, Haußmann A, Simgen A, Tomori T, Reith W, Yilmaz U. Transient in-stent stenosis: a common finding after flow diverter implantation. J Neurointerv Surg 2019;11:196-199

7. John S, Bain MD, Hui FK, Hussain MS, Masaryk TJ, Rasmussen $\mathrm{PA}$, et al. Long-term follow-up of in-stent stenosis after pipeline flow diversion treatment of intracranial aneurysms. Neurosurgery 2016;78:862-867

8. Wang T, Zhang CW, Richard SA, Chaohua W, Xie XD. Reactive in-stent stenosis of a pipeline embolization device in a child: a case report. Medicine (Baltimore) 2019;98:e18092

9. Liu Y, Shakur Y, Yoshitake M, Kambayashi Ji J. Cilostazol (pletal): a dual inhibitor of cyclic nucleotide phosphodiesterase type 3 and adenosine uptake. Cardiovasc Drug Rev 2001;19:369-386

10. Wallentin L. P2Y(12) inhibitors: differences in properties and mechanisms of action and potential consequences for clinical use. Eur Heart J 2009;30:1964-1977

11. Karan V, Vyas D, Bohra V, Huded V. Ticagrelor use in Indian patients undergoing neuroendovascular procedures: a single center experience. Neurointervention 2019;14:125-130

12. Toyoda K, Uchiyama S, Yamaguchi T, Easton JD, Kimura K, Hoshino H, et al.; CSPS.com Trial Investigators. Dual antiplatelet therapy using cilostazol for secondary prevention in patients with high-risk ischaemic stroke in Japan: a multicentre, open-label, randomised controlled trial. Lancet Neurol 2019;18:539-548

13. Stone GW, Brodie BR, Griffin JJ, Morice MC, Costantini C, St Goar FG, et al. Prospective, multicenter study of the safety and feasibility of primary stenting in acute myocardial infarction: in-hospital and 30-day results of the PAMI stent pilot trial. Primary Angioplasty in Myocardial Infarction Stent Pilot Trial Investigators. J Am Coll Cardiol 1998;31:23-30

14. Tamhane U, Meier P, Chetcuti S, Chen KY, Rha SW, Grossman $M P$, et al. Efficacy of cilostazol in reducing restenosis in patients undergoing contemporary stent based PCl: a meta-analysis of randomised controlled trials. Eurolntervention 2009;5:384-393

15. Douglas JS Jr, Holmes DR Jr, Kereiakes DJ, Grines CL, Block E, Ghazzal ZM, et al.; Cilostazol for Restenosis Trial (CREST) Investigators. Coronary stent restenosis in patients treated with cilostazol. Circulation 2005;112:2826-2832

16. Takayama K, Taoka T, Nakagawa H, Myouchin K, Wada T, Sakamoto $M$, et al. Effect of cilostazol in preventing restenosis after carotid artery stenting using the carotid wallstent: a multicenter retrospective study. AJNR Am J Neuroradio/ 2012;33:2167-2170

17. Takigawa T, Matsumaru Y, Hayakawa M, Nemoto S, Matsumura A. Cilostazol reduces restenosis after carotid artery stenting. J VasC Surg 2010;51:51-56

18. Galyfos G, Geropapas G, Sigala F, Aggeli K, Sianou A, Filis K. Meta-analysis of studies evaluating the effect of cilostazol on major outcomes after carotid stenting. J Endovasc Ther 2016;23:186195

19. Nakagawa I, Wada T, Park HS, Nishimura F, Yamada S, Nakagawa $\mathrm{H}$, et al. Platelet inhibition by adjunctive cilostazol suppresses the frequency of cerebral ischemic lesions after carotid artery stenting in patients with carotid artery stenosis. J Vasc Surg 2014,59:761-767

20. Hassan AE, Zacharatos H, Grigoryan M, Tekle WG, Khan A, Siddiq $F$, et al. Open-label phase I clinical study to assess the safety and efficacy of cilostazol in patients undergoing internal carotid artery stent placement. Interv Neurol 2017;6:42-48

21. Aguilar Pérez $M$, Bhogal $P$, Henkes E, Ganslandt $O$, Bäzner $H$, Henkes $\mathrm{H}$. In-stent stenosis after p64 flow diverter treatment. Clin Neuroradio/ 2018;28:563-568 
22. Schwartz RS, Chronos NA, Virmani R. Preclinical restenosis models and drug-eluting stents: still important, still much to learn. J Am Coll Cardiol 2004;44:1373-1385

23. Monteiro A, Lopes DK, Aghaebrahim A, Hanel R. Optical coherence tomography for elucidation of flow-diversion phenomena: the concept of endothelized mural thrombus behind reversible in-stent stenosis in flow-diverters. [published online ahead of print Mar 22, 2021] Interv Neuroradiol 2021
24. Wu CK, Lin JW, Wu LC, Chang CH. Risk of heart failure hospitalization associated with cilostazol in diabetes: a nationwide case-crossover study. Front Pharmacol 2019;9:1467

25. Zhang Z, Foster JK, Kolm P, Jurkovitz CT, Parker KM, Murrah NV, et al. Reduced 6-month resource use and costs associated with cilostazol in patients after successful coronary stent implantation: results from the Cilostazol for RESTenosis (CREST) trial. Am Heart J 2006;152:770-776 